\title{
Risk factor analysis for bone marrow histiocytic hyperplasia with hemophagocytosis: an autopsy study
}

\author{
Kunihiro Inai • Sakon Noriki • Hiromichi Iwasaki • \\ Hironobu Naiki
}

Received: 19 December 2013 /Revised: 15 April 2014 / Accepted: 11 May 2014 / Published online: 23 May 2014

(C) The Author(s) 2014. This article is published with open access at Springerlink.com

\begin{abstract}
The excessive release of inflammatory cytokines occasionally induces life-threatening hemophagocytosis referred to as hemophagocytic syndrome (HPS). A similar condition, histiocytic hyperplasia with hemophagocytosis $(\mathrm{HHH})$, is often seen in bone marrow collected during autopsy. Unlike HPS, the pathogenesis of HHH remains unclear. Therefore, we performed a clinicopathological analysis of HHH from 70 autopsy cases at the University of Fukui Hospital. HHH was detected in 29 of 70 autopsies $(41.4 \%)$ and was significantly complicated with hematological diseases $(p<0.05)$ and sepsis $(p<0.05)$. The percentage of macrophages in bone marrow (BM) nucleated cells was significantly increased in HHH $(p<0.001)$. Data from medical records indicated no significant changes, except for the minimum values of white blood cell counts $(p<0.05)$ and platelet counts $(p<0.05)$ in HHH patients as compared with non-HHH patients. Concentrations of inflammatory mediators including IL-1 $\beta$, IL-6, and IL-8 were significantly increased in $\mathrm{HHH}$ patients. Multivariate risk factor analysis identified hematological diseases (odds ratio (OR), 11.71), $\geq 15 \%$ BM macrophages (OR, 9.42), sepsis (OR, 7.77), and high serum IL-6 levels $(\mathrm{OR}, 1.00)$ as independent risk factors for $\mathrm{HHH}$. $\mathrm{HHH}$
\end{abstract}

Electronic supplementary material The online version of this article (doi:10.1007/s00428-014-1592-8) contains supplementary material, which is available to authorized users.

K. Inai $(\bowtie) \cdot H$. Naiki

Division of Molecular Pathology, Department of Pathological

Sciences, University of Fukui, Eiheiji Fukui 910-1193, Japan

e-mail: kinai@u-fukui.ac.jp

S. Noriki

Division of Tumor Pathology, Department of Pathological Sciences,

University of Fukui, Eiheiji Fukui 910-1193, Japan

H. Iwasaki

Division of Hematology and Oncology, Department of General

Medicine, University of Fukui, Eiheiji Fukui 910-1193, Japan with hypocellular BM, the most aggressive form of $\mathrm{HHH}$, was recognized in 8 of $29 \mathrm{HHH}$ patients and was associated with $\geq 25 \%$ BM macrophages $(p<0.001)$, leukocytopenia $(p<0.05)$, and high IL-8 levels $(p<0.05)$. None of the HHH patients fulfilled the diagnostic criteria of HPS. These findings suggest that HHH is a different entity from HPS and that it preferentially develops under conditions of excessive inflammation and its associated risks, such as hematological diseases and sepsis.

Keywords Bone marrow · Hematological diseases . Histiocytic hyperplasia with hemophagocytosis . Inflammation · Inflammatory cytokine $\cdot$ Sepsis

\section{Introduction}

The systemic release of excessive amounts of inflammatory cytokines results in severe clinical disorders such as acute respiratory distress syndrome, disseminated intravascular coagulation (DIC), septic shock, and multi-organ failure [1]. Simultaneously, the mononuclear-macrophage system is often dramatically activated [2], so that some patients develop a rare life-threatening syndrome called hemophagocytic syndrome (HPS) or hemophagocytic lymphohistiocytosis [3, 4]. The hallmark of HPS is sustained inflammatory cytokinemia due to genetic inheritance, infections, malignant lymphoma, and collagen diseases $[3,5,6]$. Patients with HPS develop persistent high fever, pancytopenia, hepatosplenomegaly, lymphadenopathy, and elevated levels of liver transaminases, bilirubin, lactate dehydrogenase, ferritin, and triglycerides [4, 7]. Histopathological examination reveals the presence of activated macrophages in hematopoietic organs such as bone marrow, liver, spleen, and lymph nodes, where they often engulf erythrocytes and hematopoietic nucleated cells [4]. 
Bone marrow samples from deceased patients often reveal similar findings of hemophagocytosis [2, 8]. This phenomenon, known as histiocytic hyperplasia with hemophagocytosis $(\mathrm{HHH})$, can be observed in patients with sepsis, viral and nonviral infections, and advanced malignancies $[8,9]$. Based on these observations, we hypothesized that the pathogenesis of HHH may be initiated by a series of cytokine storms including the release of inflammatory interferon (IFN)- $\gamma$, tumor necrosis factor (TNF)- $\alpha$, interleukin (IL)-1 $\beta$, IL-6, IL-10, and IL-18 cytokines, and IL- 8 chemokine, as occurs in HPS $[5,6,10]$. However, it remains controversial whether the development of HHH is caused by malignant diseases [2], and the pathogenesis of HHH remains obscure. In the present study, we investigated the pathogenesis of $\mathrm{HHH}$ by performing a postmortem analysis of 70 patients with $\mathrm{HHH}$. The primary outcome was to identify risk factors for the development of $\mathrm{HHH}$, and the secondary endpoint was to analyze the inflammatory cytokine profiles that characterize $\mathrm{HHH}$ by using serum collected at autopsy. We found that $\mathrm{HHH}$ preferentially developed under conditions of excessive inflammation, including hematological diseases such as leukemia, lymphoma, and aplastic anemia and sepsis, and that its onset and severity were associated with pro-inflammatory IL-6 and IL-8, respectively.

\section{Materials and methods}

\section{Patients and criteria for $\mathrm{HHH}$}

During the 3-year study period, we performed 87 autopsies. Seven of these cases were excluded due to the inability to collect serum because of coagulation, and ten cases were excluded because only a partial dissection was performed. The remaining 70 patients ( 39 men and 31 women) ranged in age from 0 to 94 years (median age, 68 years) at the time of death. Autopsies were performed at the University of Fukui Hospital and were retrospectively examined. Written informed consent was obtained from the families of each deceased patient prior to enrollment. All research protocols were approved by the ethics review board at the University of Fukui Hospital and conformed to the provisions of the Declaration of Helsinki.

The activity of hemophagocytosis was categorized though microscopic examination of bone marrow as follows: (a) mild $\mathrm{HHH}$, several high-power fields must be searched to find hemophagocytosis; (b) moderate HHH, 1-3 hemophagocytic cells per high-power field; (c) severe $\mathrm{HHH}$, more than 3 hemophagocytic cells per high-power field; and (d) hypoplastic HHH (hypo-HHH), severe HHH with hypocellular bone marrow. We assumed that cases showing moderate HHH to hypo-HHH represented HHH in accordance with previously described criteria $[2,8]$.

Lists of inspection data

Data including age, sex, diagnosis on admission, complications, DIC, infectious disease, sepsis, duration until autopsy, and cause of death were obtained from clinical and autopsy records (Electronic supplementary material (ESM) Table 1). Mean duration until autopsy was $2.9 \pm 2.6 \mathrm{~h}(1-15.5 \mathrm{~h}$; median duration, $2 \mathrm{~h}$ ) after the patient's death. This time was similar in cases with $\mathrm{HHH}(3.4 \pm 3.3 \mathrm{~h})$ and with non-HHH $(2.7 \pm 2.0 \mathrm{~h})$.

Table 1 Patient characteristics with or without $\mathrm{HHH}$

\begin{tabular}{|c|c|c|c|c|c|}
\hline Category & Subcategory & Total & HHH & No HHH & $P^{*}$ \\
\hline Number of patients & & 70 & 29 & 41 & \\
\hline \multirow[t]{3}{*}{ Age } & Average & 66.5 & 65.4 & 66.9 & \\
\hline & Range & $0-94$ & $52-91$ & $0-94$ & \\
\hline & Median & 68 & 65 & 71 & \\
\hline \multirow[t]{2}{*}{ Sex } & Male & 39 & 16 & 23 & \\
\hline & Female & 31 & 13 & 18 & \\
\hline Hematological diseases & & 19 & 14 & 5 & $<0.05$ \\
\hline \multirow[t]{3}{*}{ Malignancy } & All & 49 & 22 & 27 & \\
\hline & Hematological & 18 & 13 & 5 & $<0.05$ \\
\hline & Solid & 31 & 9 & 22 & \\
\hline \multirow[t]{3}{*}{ Infection } & All & 42 & 22 & 20 & \\
\hline & Sepsis & 18 & 13 & 5 & $<0.05$ \\
\hline & Pneumonia & 16 & 12 & 14 & \\
\hline DIC & & 32 & 18 & 14 & \\
\hline Shock & & 17 & 9 & 8 & \\
\hline
\end{tabular}

$H H H$ histiocytic hyperplasia with hemophagocytosis, DIC disseminated intravascular coagulation

*Adjusted using the Holm method for multiple testing 
Sepsis was verified by the repeated detection of microbes from ante-mortem blood cultures from patients who met the criteria of systemic inflammatory response syndrome $[11,12]$ or by the detection of scattered micro-abscesses with or without bacterial, fungal, or viral inclusion bodies in histopathological studies of different tissues. DIC was diagnosed based on the criteria of the Japanese Ministry of Health, Labor and Welfare [13] or from the existence of fibrin thrombi in tissue sections collected during autopsy. Shock was pathologically confirmed by detecting shock-related morphological changes including diffuse alveolar damage (shock lung), scattered focal or submassive necrosis around the central hepatic vein (shock liver), and/or acute tubular necrosis (shock kidney) [1]. Hepatomegaly was defined as a liver weight greater than $1,300 \mathrm{~g}$ (normal range in Japanese people, 900-1,300 g), and splenomegaly was defined as a spleen weight of $130 \mathrm{~g}$ or greater.

The maximum and minimum white blood cell (WBC) counts, minimum hemoglobin $(\mathrm{Hb})$ level and platelet count (Plt), and peak serum values of aspartate aminotransferase (AST), triglyceride, ferritin, and soluble IL-2 receptor (sIL2R) from within 100 days prior to death were collected from medical records.

Morphological analysis and immunohistochemical staining

Thin sections of paraffin-embedded bone marrow were stained with hematoxylin and eosin. Activated macrophages were evaluated by immunohistochemical staining with primary anti-CD68 antibody (DAKO, Kyoto, Japan) and antibody against galactin-3 (Nichirei, Tokyo, Japan), a carbohydratebinding lectin secreted by activated macrophages [14]. A rabbit polyclonal anti-CD3 antibody (DAKO) was used to determine the number of T lymphocytes. The Berlin blue stain was performed to detect intra- and extracellular iron deposition [8]. The sections were evaluated by two different pathologists who specialize in the pathophysiological analysis of infection and inflammatory diseases $[15,16]$.

\section{Cytokine analysis}

Blood samples were collected from the right atrium by insertion of an $18-\mathrm{G}$ needle attached to a $20-\mathrm{ml}$ syringe after resection of the ribs and cardiac sac at autopsy. After clotting, the blood was spun at $1,000 \times g$ for $10 \mathrm{~min}$ at room temperature. The separated serum was transferred to $1.5-\mathrm{ml}$ tubes and stored at $-80{ }^{\circ} \mathrm{C}$ until analysis. The levels of inflammatory TNF- $\alpha$, IL-1 $\beta$, IL- 6 , IL-10, and IL-12 cytokines, and IL-8 chemokine were determined by flow cytometry (FACs Calibur; Becton Dickinson, Franklin Lakes, NJ) performed with a Human Inflammation Kit (BD Biosciences, San Diego, CA) according to the manufacturer's protocol. IFN- $\gamma$ was measured by using a BD cytometric bead array (Becton Dickinson) as described before [17]. If the measured levels exceeded the upper limits of the equipment, serum was diluted with saline and then re-evaluated by the same method.

Statistical analysis

Statistical analysis was performed by using the $\chi^{2}$ test and Mann-Whitney $U$ test. Pearson and Spearman correlation coefficients were calculated as appropriate. Significant associations with $\mathrm{HHH}$ were further analyzed by using logistic regression analysis. $P$ values were adjusted by using the Holm method for multiple testing [18], and the results before and after the adjustment are shown in ESM Tables 2 and 3. Values of $p<0.05$ were accepted as statistically significant. All statistical analyses were performed with Ekuseru-Toukei 2010 software (Social Survey Research Information, Tokyo, Japan).

\section{Results}

Patient characteristics with $\mathrm{HHH}$

Twenty-nine of 70 patients $(41.4 \%)$ were categorized as having HHH. The HHH group consisted of high-grade cases demonstrating moderate HHH $(n=10)$, severe HHH $(n=11)$, or hypo-HHH $(n=8)$. The remaining 41 patients categorized as having no $\mathrm{HHH}$ consisted of 14 patients with mild $\mathrm{HHH}$ and 27 normal cohorts. Bone marrow cellularity of the 70 patients was characterized as hypercellular $(n=26)$, slightly hypercellular $(n=3)$, normocellular $(n=28)$, slightly hypocellular $(n=1)$, and hypocellular $(n=12)$. Representative morphological features are shown in Fig. 1. CD68-expressing cells were scattered throughout all bone marrow sections. In particular, severe HHH and hypo-HHH cases contained large amebic histiocytes expressing both CD68 and galectin-3 proteins; these cells were considered to represent activated macrophages. The cytoplasm of many activated cells contained engulfed erythrocytes, nucleated cells, and/or vacuoles. The percentage of CD68-positive cells in the bone marrow nucleated cell number was significantly increased in patients with $\mathrm{HHH}$ than in patients without HHH $(25.2 \% \pm 20.0 \%$ vs. $12.1 \% \pm 4.0 \%, p<0.001$ ) (Fig. 2). Clinical characteristics of patients with or without HHH are shown in Table 1. Hematological diseases $(p<0.05)$, hematological malignancies $(p<0.05)$, and sepsis $(p<0.05)$ were significantly more common in patients with $\mathrm{HHH}$ than in patients without $\mathrm{HHH}$. Malignancies, pneumonia, and shock were not associated with $\mathrm{HHH}$.

Sepsis as a critical cause of death in $\mathrm{HHH}$

To clarify the characteristic causes of death in $\mathrm{HHH}$, the causes of death were categorized (Table 2). The following causes of death were identified in the $29 \mathrm{HHH}$ patients: acute 


\section{Normocellular} bone marrow

\section{HE stain LPF}
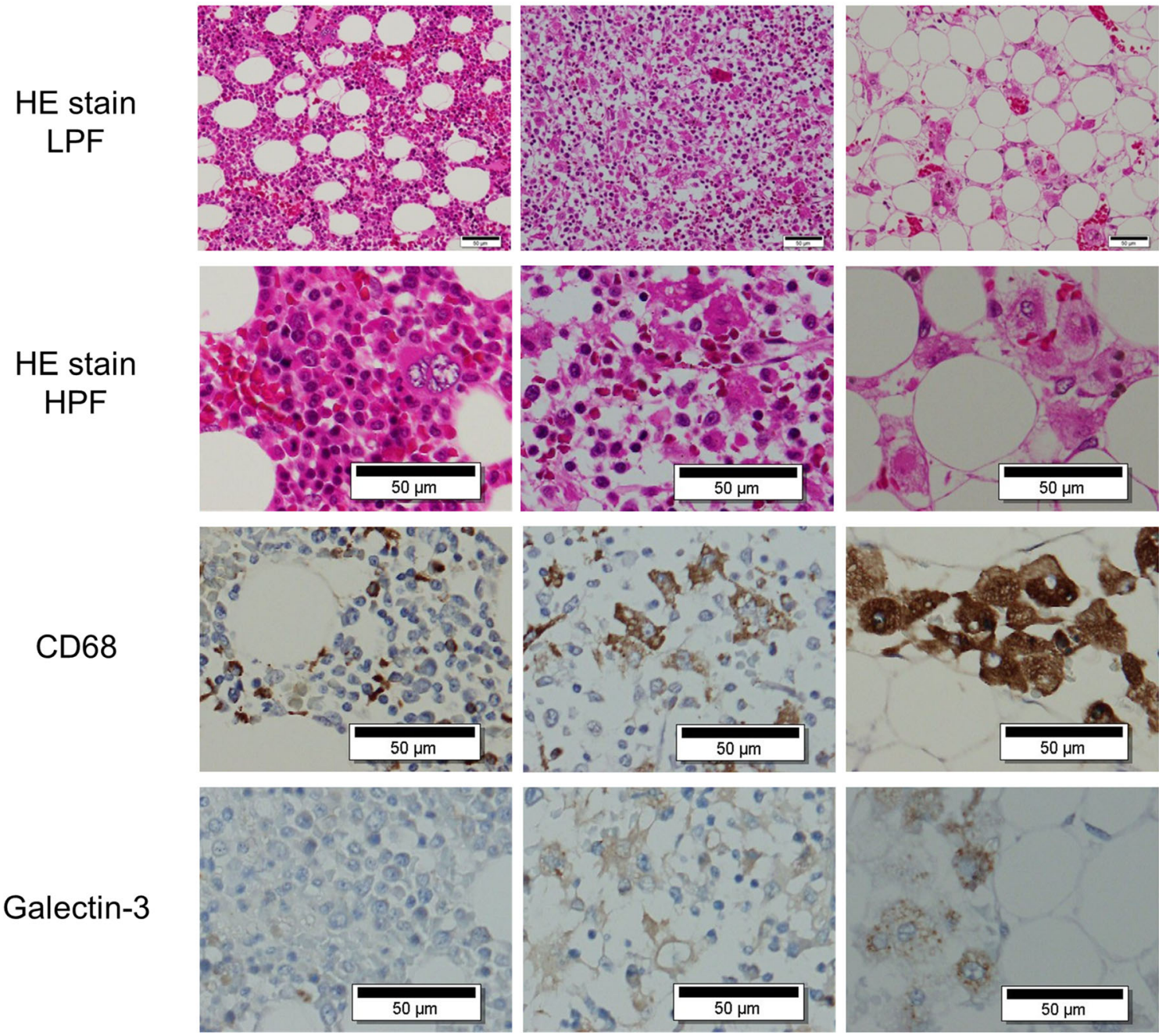

Fig. 1 Morphological features of bone marrow at autopsy in patients with HHH. (Top line) Low-power magnification of bone marrow stained by hematoxylin and eosin. (2nd line) High-power magnification of HEstained bone marrow. Activated macrophages engulfing erythrocytes and nucleated cells were scattered in severe HHH (center column) and hypo$\mathrm{HHH}$ (right column) sections. (3rd line) Immunohistochemical staining with anti-CD68 antibody. Although CD68-positive macrophages, including brown deposits in the cytoplasm, were present in all bone marrow tissues, large amoebic-shaped cells were only noted in severe $\mathrm{HHH}$ and hypo-HHH bone marrow. (Bottom line) Immunohistochemical staining of galectin-3 secreted by active macrophages. Galectin-3 was expressed in bone marrow from severe $\mathrm{HHH}$ and hypo-HHH patients. $H H H$, histiocytic hyperplasia of hemophagocytosis; $H E$, hematoxylin and eosin; $L P F$, low-power field; $H P F$, high-power field respiratory distress syndrome (ARDS)/sepsis/septic shock $(n=11)$, cancer $(n=6)$, organ failure such as congestive heart failure or liver failure $(n=6)$, pneumonia $(n=3)$, and shock other than septic shock $(n=3)$. Organ failure was the predominant cause of death in non-HHH patients, followed by shock and cancer. Death caused by sepsis/septic shock or ARDS occurred in only two non-HHH cases. The percentage was significantly lower than that of HHH cases (2/41 vs. 11/29, $p<0.05$ ).

The pathogens isolated by microscopic inspection from septic HHH patients were fungi $(n=6$; Candida in three patients, Aspergillus in two patients, and Fusarium in one patient), cocci $(n=6)$, bacilli ( $n=3)$, and cytomegaloviruses $(\mathrm{CMV})(n=3)$. Three different pathogens (fungus, CMV, and cocci) were isolated from two patients, and a female patient with microscopic polyangiitis had both Candida and Aspergillus infections. In contrast to $\mathrm{HHH}$ patients, three patients with cocci and one patient with bacilli were found among the 41 non-HHH patients.

High serum inflammatory cytokine levels in $\mathrm{HHH}$

HPS is induced by the simultaneous release of cytokines such as IFN- $\gamma$, TNF- $\alpha$, IL-6, IL-1 $\beta$, IL-10, IL-12, IL-8, and 


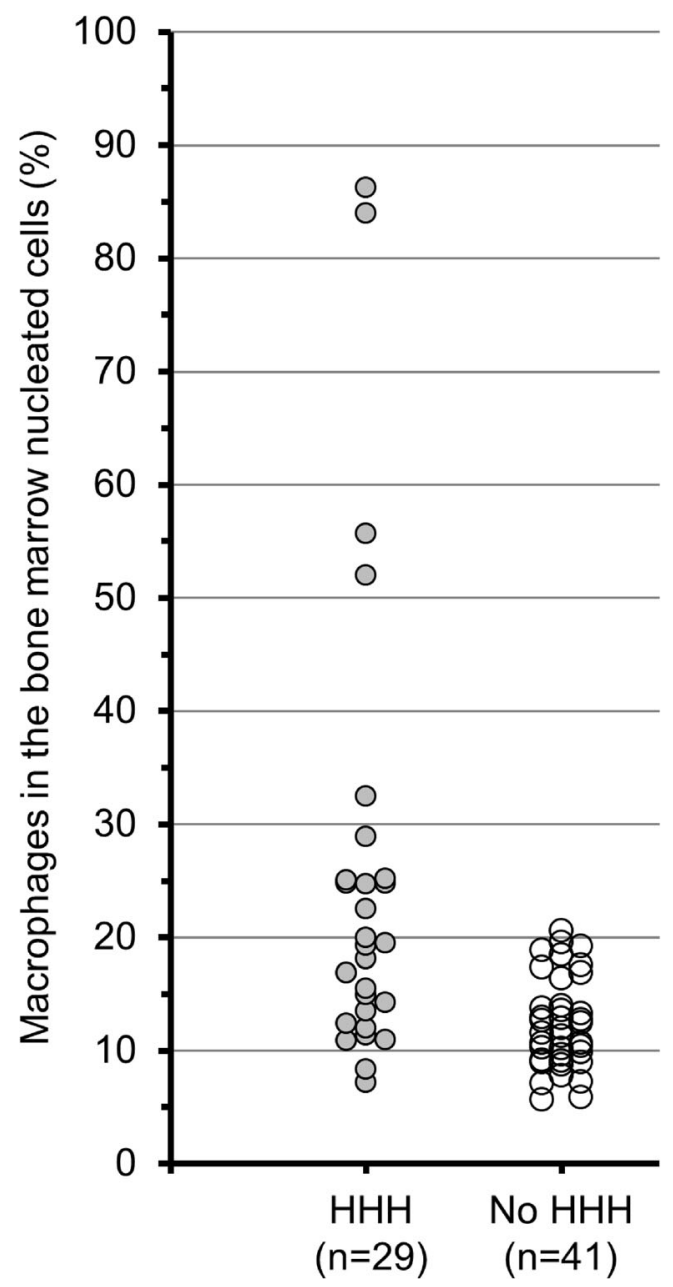

Fig. 2 Percentage of macrophages in the bone marrow nucleated cells. The macrophages in the bone marrow were evaluated by CD68 immunohistochemical stain. Bone marrow nucleated cells were counted as Giemsa-staining positive cells. $H H H$, histiocytic hyperplasia of hemophagocytosis

macrophage-colony stimulating factor [19]. In this study, IL-6 $(20,662.2 \pm 28,447.0 \mathrm{pg} / \mathrm{ml}$ vs. $4,262.5 \pm 11,769.6 \mathrm{pg} / \mathrm{ml}$, $p<0.001)$, IL-1 $\beta(749.8 \pm 2,383.8 \mathrm{pg} / \mathrm{ml}$ vs. $95.5 \pm 561.1 \mathrm{pg} /$ $\mathrm{ml}, \mathrm{p}<0.05)$, and IL-8 $(24,439.7 \pm 42,733.5 \mathrm{pg} / \mathrm{ml}$ vs. $577.0 \pm$ $816.1 \mathrm{pg} / \mathrm{ml}, p<10^{-6}$ ) levels were significantly increased in $\mathrm{HHH}$ patients as compared with patients without $\mathrm{HHH}$, while IL-12, IFN- $\gamma$, TNF- $\alpha$, and IL-10 concentrations showed no

Table 2 Cause of the patient's death with or without $\mathrm{HHH}$

\begin{tabular}{lll}
\hline & HHH & No HHH \\
\hline ARDS/sepsis/septic shock & 11 & 2 \\
Pneumonia/other infection & 3 & 4 \\
Other shock/bleeding & 3 & 11 \\
Cancer death/cachexia/emaciation & 6 & 10 \\
Organ failure/heart, respiratory, liver, or renal & 6 & 13
\end{tabular}

$H H H$ histiocytic hyperplasia with hemophagocytosis, $A R D S$ acute respiratory distress syndrome statistically significant differences between the HHH and nonHHH groups (Fig. 3a, Table 3, and ESM Table 2).

Correlations among serum cytokine levels were also analyzed (Fig. 3b). In HHH patients, TNF- $\alpha$ was significantly correlated with IL-10 $\left(r^{2}=0.819, p<10^{-10}\right)$, IL-1 $\beta\left(r^{2}=0.679\right.$, $\left.p<10^{-7}\right)$, and IL-8 $\left(r^{2}=0.530, p<10^{-5}\right)$. Correlations were also detected between IL-10 and IL-1 $\beta\left(r^{2}=0.599, p<10^{-6}\right)$, IL-10 and IL-8 $\left(r^{2}=0.576, p<10^{-5}\right)$, and IL-1 $\beta$ and IL- $8\left(r^{2}=\right.$ $\left.0.630, p<10^{-6}\right)$.

Multivariate risk factor analysis for $\mathrm{HHH}$

To elicit the critical risk factors for $\mathrm{HHH}$, logistic regression analysis was performed with variables that were significant in the univariate analysis. The presence of hematological diseases was associated with the development of HHH (odds ratio $=11.71 ; 95 \%$ confidence interval $(\mathrm{CI}), 1.83-73.93$, $p<0.01$ ) (Table 4). Other factors associated with increased risk include $15 \%$ or more of bone marrow macrophages (odds ratio $=9.42 ; 95 \% \mathrm{CI}, 2.14-41.58, p<0.01$ ), sepsis (odds ratio $=7.77$; $95 \%$ CI, $1.48-40.9, p<0.05)$, and high serum IL-6 levels (odds ratio $=1.00 ; 95 \% \mathrm{CI}, 1.00-1.0001, p<0.05$ ).

Pathophysiological features of hypo-HHH

Most HPS patients have pancytopenia, which makes them susceptible to becoming immunocompromised. However, hypocellular bone marrow is rare in HPS patients, and there have been no previous reports of HHH with marrow hypoplasia. In the present study, we found eight HHH patients with both pancytopenia and hypocellular bone marrow. We therefore investigated the characteristics of patients with hypoHHH (Table 5 and ESM Table 3). The absolute number of CD68-positive macrophages in the bone marrow was in the same range in each category (Fig. $4 \mathrm{a}$ ). The percentage of macrophages in the bone marrow was significantly increased in hypo-HHH as compared with other types of HHH and nonHHH (Fig. 4b). Interestingly, the rate was similar between severe-HHH and moderate-HHH, and between mild-HHH and no-HHH. Thus, we compared the characteristics of hypo-HHH, other-HHH (including severe- and moderate$\mathrm{HHH}$ ), and no HHH with both mild-HHH and normal cohorts. As shown in Fig. 4c, seven of eight hypo-HHH patients had more than $25 \%$ macrophages in the bone marrow. The hypoHHH group had dramatically decreased minimum WBC counts $\left(0.6 \pm 0.7 \times 10^{3} / \mu \mathrm{l}\right)$. Additionally, the IL- 8 concentration was significantly increased in hypo-HHH patients as compared with other HHH patients $(57,155.8 \pm 62,993.6 \mathrm{pg} / \mathrm{ml} \mathrm{vs}$. $11,976.4 \pm 24,010.6 \mathrm{pg} / \mathrm{ml}, p<0.01$, Fig. $4 \mathrm{c})$. Six of the eight hypo-HHH patients received chemo-radiotherapies consisting of allogeneic peripheral blood stem cell transplantation $(n=2)$, chemotherapy $(n=3)$, and splenic irradiation $(n=1)$ (Table 6). The mean duration from the end of the final therapy to death 
Fig. 3 Serum cytokine analyses in patients with $\mathrm{HHH}$. a Serum cytokine levels in patients with $\mathrm{HHH}$. Serum was collected from the right atrium of each deceased patient and stored at $-80{ }^{\circ} \mathrm{C}$ until analysis. The cytokine levels were measured by flow cytometry. Grey bars represent data derived from $\mathrm{HHH}$ patients, and open bars represent data from non$\mathrm{HHH}$ patients. The data are shown by a box and whisker plot. $X$ 's indicate statistical outliers. b Correlation coefficients among inflammatory cytokines and chemokines in $\mathrm{HHH}$ patients. Data represent the square of the correlation coefficient value. *, $* *, * * *, * * * *$, and $* * * * *$ indicate statistically significant differences with $p$ values $<0.01,<10^{-5}$, $<10^{-6},<10^{-7}$, and $<10^{-10}$, respectively. $\mathrm{HHH}$, histiocytic hyperplasia of hemophagocytosis; $n s$, not significant

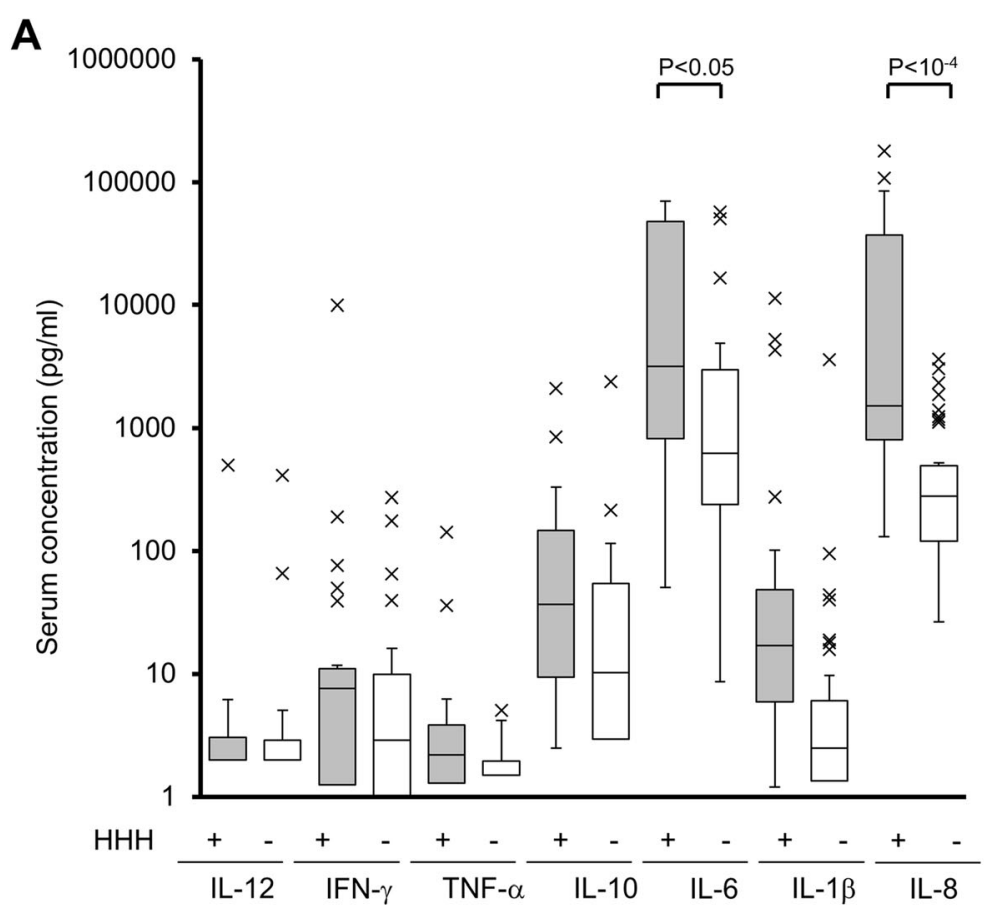

B

\begin{tabular}{|c|c|c|c|c|c|c|}
\hline $\mathrm{HHH}$ & $\mathrm{IFN}-\gamma$ & $\mathrm{TNF}-\alpha$ & $\mathrm{IL}-10$ & $\mathrm{IL}-6$ & $\mathrm{IL}-1 \beta$ & $\mathrm{IL}-8$ \\
\hline $\mathrm{IL}-12$ & $\mathrm{~ns}$ & $\mathrm{~ns}$ & $\mathrm{~ns}$ & $\mathrm{~ns}$ & $\mathrm{~ns}$ & $\mathrm{~ns}$ \\
\hline & $\mathrm{IFN}-\gamma$ & $\mathrm{ns}$ & $\mathrm{ns}$ & $\mathrm{ns}$ & $\mathrm{ns}$ & $\mathrm{ns}$ \\
\hline & $\mathrm{TNF}-\alpha$ & $0.891^{* * * * *}$ & $\mathrm{~ns}$ & $0.679^{* * * *}$ & $0.530^{* *}$ \\
\cline { 3 - 7 } & & $\mathrm{IL}-10$ & $\mathrm{~ns}$ & $0.599^{* * *}$ & $0.576^{* *}$ \\
\cline { 3 - 7 } & & & $\mathrm{IL}-6$ & $\mathrm{~ns}$ & $\mathrm{~ns}$ \\
\hline
\end{tabular}

was $79.8 \pm 94.7$ days (range, 20-270 days). Two of the eight hypo-HHH patients had not received myeloablative therapies, suggesting that the influence of chemo-radiotherapies was limited to the initiation of hypo-HHH.

Regarding CD3 immunostaining and iron staining of bone marrow samples, the Fe staining detected hemophagocytic histiocytes. However, no significant association was obtained between the number of $\mathrm{CD} 3$-positive $\mathrm{T}$ lymphocytes and the severity of $\mathrm{HHH}$ or between the Fe-positive macrophage counts and the degree of HHH (ESM Figures 1 and 2).

\section{Discussion}

HPS, which is characterized by the proliferation of activated macrophages with hemophagocytosis, is a rare syndrome $[4$,
$6,7,20]$. In contrast, HHH was detected in $41.4 \%$ of this study cohort. Studies performed at two different university hospitals identified HHH in 35 of 107 deceased patients (32.7\%) [8] and 102 of 230 autopsies (44.3\%) [2]. In addition, the patients with mild HHH form the largest cohort in these previous studies. These data are consistent with our results, and together, they suggest that $\mathrm{HHH}$ is a common phenomenon in patients who die in major hospitals.

Two major categories of risk factors for HHH have been identified: (i) intrinsic factors, such as advanced malignancies, sepsis, and multi-organ failure; and (ii) extrinsic factors, such as blood transfusions and treatment intensity [2, 8, 21]. However, some controversial results have been obtained. In comparison with non-HHH patients, HHH patients had a significantly increased frequency of hematological diseases, hematological malignancies, and sepsis. Independent risk factors 
Table 3 Clinical and physiological findings of patients with or without $\mathrm{HHH}$

\begin{tabular}{llll}
\hline & HHH $(n=29)$ & No HHH $(n=41)$ & $\begin{array}{l}P^{\mathrm{a}} \\
\text { value }\end{array}$ \\
\hline Organomegaly & & & \\
Hepatomegaly & $16(55.2 \%)$ & $15(36.6 \%)$ & \\
Splenomegaly & $16(55.2 \%)$ & $12(29.3 \%)$ & \\
Laboratory data & & & \\
WBC max (x10 $\left.{ }^{3} / \mu \mathrm{l}\right)$ & $18.5 \pm 13.6$ & $17.4 \pm 8.9$ & \\
WBC min $\left(\mathrm{x} 10^{3} / \mu \mathrm{l}\right)$ & $4.3 \pm 7.4$ & $5.7 \pm 3.3$ & $<0.05$ \\
Hb (g/dl) & $7.2 \pm 2.4$ & $8.1 \pm 2.5$ & \\
Plt (x10 $/ \mu \mathrm{l})$ & $5.7 \pm 10.8$ & $11.6 \pm 9.7$ & $<0.05$ \\
AST $(\mathrm{IU} / \mathrm{l})$ & $147 \pm 170$ & $766 \pm 2,807$ & \\
Triglyceride (mg/dl) & $239 \pm 244$ & $147 \pm 89$ & \\
IL-12 (pg/ml) & $19.0 \pm 92.2$ & $13.4 \pm 64.4$ & \\
IFN- $\gamma(\mathrm{pg} / \mathrm{ml})$ & $361.2 \pm 1,854.2$ & $17.3 \pm 50.0$ & \\
TNF- $\alpha(\mathrm{pg} / \mathrm{ml})$ & $8.4 \pm 26.7$ & $1.4 \pm 1.2$ & \\
IL-10 (pg/ml) & $172.8 \pm 407.8$ & $78.9 \pm 372.5$ & \\
IL-6 (pg/ml) & $20,662.2 \pm$ & $4,262.5 \pm$ & $<0.05$ \\
IL-1 $\beta(\mathrm{pg} / \mathrm{ml})$ & $28,447.0$ & $11,769.6$ & $<0.05$ \\
IL-8 (pg/ml) & $24,439.7 \pm$ & $577.0 \pm 816.1$ & $<10^{-4}$ \\
& $42,733.5$ & & \\
Macrophages in BM & $25.2 \pm 20.0$ & $12.1 \pm 4.0$ & $<001$ \\
cells $(\%)$ & & & \\
\hline
\end{tabular}

$H H H$ histiocytic hyperplasia with hemophagocytosis, WBC max maximum number of WBC, $W B C$ min minimum number of WBC

${ }^{a}$ Adjusted using the Holm method for multiple testing

for HHH identified by logistic regression analysis were hematological diseases, $\geq 15 \%$ bone marrow macrophages, sepsis, and high IL-6 levels. Hematological diseases were the most striking risk factor, and they have been implicated in severe immunodeficiency. Nevertheless, an underlying immunodeficient state at the onset of HHH and HPS seems underrepresented, unlike the documented infections for the most common setting of secondary HPS [22]. However, HHH developed preferentially in our hematological subjects with significantly decreased nadir WBC counts, particularly in

Table 4 Multivariate risk analysis for $\mathrm{HHH}$

\begin{tabular}{llll}
\hline & Odds ratio & $95 \% \mathrm{CI}$ & $P$ value \\
\hline Hematological diseases & 11.71 & $1.83-74.93$ & $<0.01$ \\
Sepsis & 7.77 & $1.48-40.9$ & $<0.05$ \\
WBC (min) & 1.12 & $0.94-1.33$ & 0.20 \\
IL-6 & 1.00 & $1.00-1.0001$ & $<0.05$ \\
BM macrophages $(\geq 15 \%)$ & 9.42 & $2.14-41.58$ & $<0.01$
\end{tabular}

$H H H$ histiocytic hyperplasia with hemophagocytosis, $W B C$ (min) minimum number of WBC, $B M$ bone marrow, $C I$ confidence interval
Table 5 Features of patients with hypo-HHH or other HHH

\begin{tabular}{llll}
\hline & $\begin{array}{l}\text { Hypo-HHH } \\
(n=8)\end{array}$ & $\begin{array}{l}\text { Other HHH } \\
(n=21)\end{array}$ & $\begin{array}{l}P^{\mathrm{a}} \\
\text { value }\end{array}$ \\
\hline Hematological diseases & $7(87.5 \%)$ & $7(33.3 \%)$ & \\
$\begin{array}{l}\text { BM macrophages } \\
\quad(25 \%)\end{array}$ & $7(87.5 \%)$ & $1(4.8 \%)$ & $<0.001$ \\
WBC max $\left(\times 10^{3} / \mu \mathrm{l}\right)$ & $17.9 \pm 16.6$ & $18.7 \pm 12.8$ & \\
WBC $\min \left(\times 10^{3} / \mu \mathrm{l}\right)$ & $0.6 \pm 0.7$ & $5.8 \pm 8.2$ & $<0.05$ \\
Hb $(\mathrm{g} / \mathrm{dl})$ & $6.1 \pm 1.2$ & $7.6 \pm 2.6$ & \\
Plt $\left(\times 10^{4} / \mu \mathrm{l}\right)$ & $1.1 \pm 1.1$ & $7.5 \pm 12.4$ & \\
Triglyceride $(\mathrm{mg} / \mathrm{dl})$ & $408 \pm 315$ & $155 \pm 150$ & \\
IL-12 $(\mathrm{pg} / \mathrm{ml})$ & $1.8 \pm 1.4$ & $25.6 \pm 108.3$ & \\
IFN- $\gamma(\mathrm{pg} / \mathrm{ml})$ & $33.4 \pm 65.8$ & $486.0 \pm 2,780.0$ & \\
TNF- $\alpha(\mathrm{pg} / \mathrm{ml})$ & $20.6 \pm 3.8$ & $49.5 \pm 7.6$ & \\
IL-10 $(\mathrm{pg} / \mathrm{ml})$ & $472.9 \pm 708.8$ & $58.5 \pm 85.3$ & \\
IL-6 $(\mathrm{pg} / \mathrm{ml})$ & $29,865.0 \pm$ & $17,156.4 \pm$ & \\
& $33,572.3$ & $26,299.4$ & \\
IL-1 $\beta(\mathrm{pg} / \mathrm{ml})$ & $2,005.6 \pm 4061.3$ & $271.6 \pm 1,145.6$ & \\
IL-8 $(\mathrm{pg} / \mathrm{ml})$ & $57,155.8 \pm$ & $11,976.4 \pm$ & $<0.05$ \\
& $62,993.6$ & $24,010.6$ & \\
\hline
\end{tabular}

$H H H$ histiocytic hyperplasia with hemophagocytosis, WBC $\max$ maximum number of WBC, WBC min minimum number of WBC

${ }^{\text {a }}$ Adjusted using the Holm method for multiple testing

hematological patients with hypo-HHH. Hematological patients with leukemia, malignant lymphoma, myelodysplastic syndrome, or aplastic anemia are potentially immunosuppressed, which would make them susceptible to severe infections such as pneumonia, febrile neutropenia, and bloodstream infections [15, 23]. Hematological disorder itself is not a condition of excessive inflammation; however, its associated risk of infections may lead to inflammation. The importance of severe infection for HHH may be validated by the other independent risk factors of sepsis and high IL-6 levels. In fact, sepsis is defined as the systemic response to infection and is involved in the systemic inflammatory response syndrome $[11,12]$. IL-6 concentrations are proportional to the severity of infection [24]. The kinetics of inflammatory mediators after death remains unclear. Indeed, few studies have provided sequential analyses of inflammatory cytokine levels after death. As for the IL- 6 and IL- $1 \beta$ concentrations, the mean levels tend to gradually increase at $24 \mathrm{~h}$ after death in both septic patients and normal cohorts, but several cases have shown decreased levels after death $[25,26]$. Because our autopsies were performed within $24 \mathrm{~h}$ of death $(3.0 \pm 2.6 \mathrm{~h}$; range, $1-15.5 \mathrm{~h}$ ), postmortem modifications are assumed to be slight. The results of previous studies together with the present results suggest that underlying diseases characterized by excessive inflammatory conditions are the most striking initiators of $\mathrm{HHH}$.

The median time from onset to diagnosis of hemophagocytic lymphohistiocytosis is 3.5 months [19], and 
Fig. 4 Features of hypo-HHH. a Absolute number of CD68positive macrophages in the bone marrow in different types of HHH. b Percentage of macrophages in the bone marrow nucleated cells in each category. Grey bars represent data derived from $\mathrm{HHH}$ patients, and open bars represent data from non$\mathrm{HHH}$ patients. The data are shown by a box and whisker plot. $X$ 's indicate statistical outliers. c Characteristics of hypo-HHH in comparison with other-HHH and non-HHH patients. Closed bars represent data from hypo-HHH patients, oblique bars represent data from $\mathrm{HHH}$ patients, and open bars represent data from non-HHH patients. Data are presented as the mean \pm standard deviation. WBC min, minimum number of white blood cells; $H H H$, histiocytic hyperplasia of hemophagocytosis; hypo-HHH, hypoplastic $\mathrm{HHH}$
A

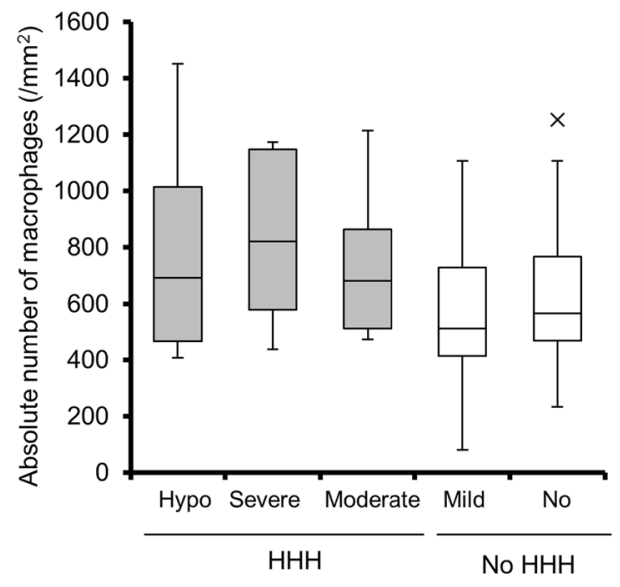

C

Patients with $\geq 25 \%$ macrophages In the BM nucleated cells
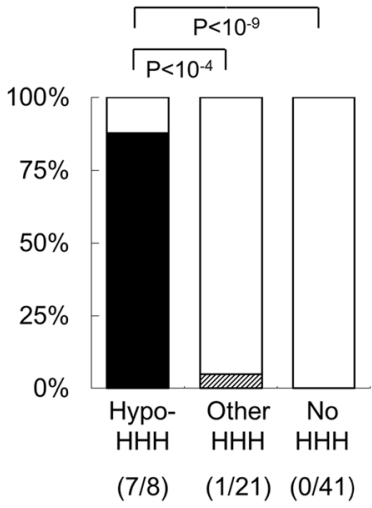

B

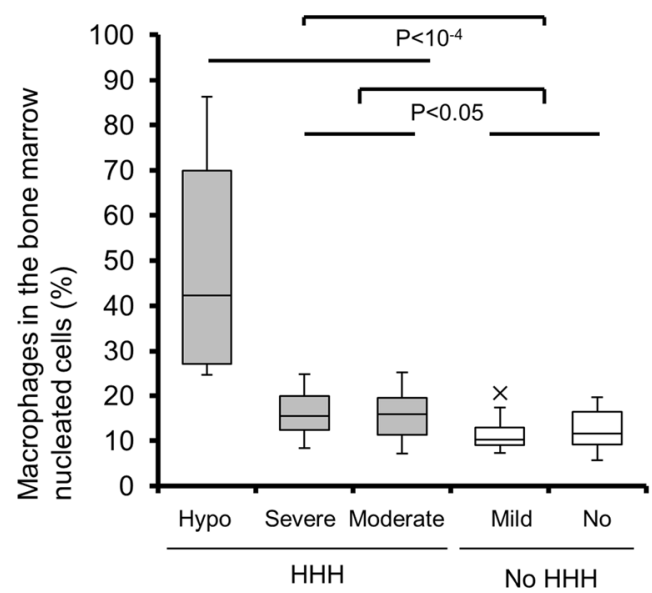

the activated inflammatory cytokines often persist for 100 days [5]. In addition, the impairment of the Th1 and Th2 balance promotes secretion of Th1 cytokines such as IFN- $\gamma$, IL-4, IL12 , and IL-18, followed by the elevation of pro-inflammatory TNF- $\alpha$, IL- $1 \beta$, and IL- 6 cytokines, IL- 8 chemokine, and antiinflammatory IL-10 cytokine [5, 10, 19]. However, the profiles of inflammatory cytokines have not been previously analyzed in $\mathrm{HHH}$ patients. We found that $\mathrm{HHH}$ patients had significantly higher levels of IL-1 $\beta$, IL-6, and IL-8 as compared with patients without $\mathrm{HHH}$. Several mediators including TNF- $\alpha$, IL-1 $\beta$, IL-8, and IL-10 showed strong positive correlations, indicating that the secreted cytokines were closely associated. The released cytokines were similar to the cytokine profiles described for septic shock, severe febrile neutropenia, and in vitro sepsis model experiments that used peripheral blood monocytes [27-29]. Moreover, significant correlations are noted among inflammatory cytokines in severe inflammatory diseases [28, 29]. These findings suggest that a series of inflammatory cytokine releases was a feature of
$\mathrm{HHH}$ as well as other severe inflammatory diseases and that monocytes and macrophages were responsible for the production of these cytokines. On the other hand, the levels of IFN- $\gamma$ did not show a statistically significant difference between the $\mathrm{HHH}$ and non-HHH patients. The levels of IFN- $\gamma$ decrease rapidly with improvements in the HPS syndrome [5, 19], suggesting that the IFN- $\gamma$ concentration had already attenuated at the time of death.

$\mathrm{HHH}$ patients have been reported to have normocellular bone marrow, but we identified eight $\mathrm{HHH}$ patients with hypocellular bone marrow. Hypo-HHH was characterized by an increased rate of migrated macrophages in the bone marrow, severe leukocytopenia, and high serum IL-8 levels; however, there was no increase in the absolute number of macrophages, indicating that the functional activation of macrophages resulted in the engulfment of bone marrow hematopoietic cells. High IL- 8 concentrations may be associated with the seriousness of $\mathrm{HHH}$. Indeed, the chemokine concentration was significantly increased according to the severity of $\mathrm{HHH}$. 
Table 6 Days after the final chemo-radiotherapy of patients with hypo-HHH

\begin{tabular}{llll}
\hline No & Clinical diagnosis & Days after the final chemo-radiotherapy & Regimen \\
\hline 1 & AML & 51 days & CPA, TBI (Allo-PBSCT) \\
2 & AML & 23 days & CPA, TBI (Allo-PBSCT) \\
3 & AML & 55 days & Ara-C, MIT, VP-16 \\
4 & Malignant lymphoma & 20 days & Ara-C, VP-16, DEX \\
5 & Malignant lymphoma & 60 days & L-ASP \\
6 & Myelofibrosis & 270 days & Splenic irradiation \\
7 & Aplastic anemia & Not done & \\
\hline
\end{tabular}

Allo-PBSCT allogeneic peripheral blood stem cell transplantation, $A M L$ acute myelocytic leukemia, $A r a-C$ cytarabine, $C P A$ cyclophosphamide, $D E X$ dexamethasone, MIT mitoxantrone hydrochloride, $L-A S P$ L-asparaginase, TBI total body irradiation, $V P$ - 16 etoposide

Even in the patients with mild HHH, the IL-8 concentration was greater than that in the normal cohort $(714.2 \pm 649.0 \mathrm{pg} / \mathrm{ml}$ vs. $505.8 \pm 893.7 \mathrm{pg} / \mathrm{ml}, p<0.05)$, although their macrophage counts were in the same range. Conversely, the minimum WBC count of mild $\mathrm{HHH}$ was significantly lower than that of non-HHH $\left(4.2 \pm 2.9 \times 10^{3} / \mu 1\right.$ vs. $\left.6.5 \pm 3.2 \times 10^{3} / \mu 1, p<0.05\right)$. IL- 8 is a CXC chemokine produced by monocytes and macrophages that attracts neutrophils to inflamed sites for phagocytosis and killing of pathogens [27, 30]. The IL-8 concentrations are inversely correlated with leukocytopenia, and the peak value is seen during the neutropenic phase of hematopoietic stem cell transplantation [31]. These findings suggest that the high IL-8 level in hypo-HHH is the consequence of severe leukocytopenia due to excessive activation of bone marrow macrophages.

Strauss et al. described HPS as the most severe variant of HHH [8]. However, none of the HHH patients in their study or in our study fulfills the diagnostic criteria for HPS. Indeed, HPS

Table 7 A tentative definition of "polyhemphagocytosis (PHP)"

\begin{tabular}{lccc}
\hline Prerequisite condition & Inai & Strauss & Suster \\
\hline $\begin{array}{l}\geq 1-3 \text { hemophagocytic cells/HPF in BM, } \\
\text { lymphocytes, or spleen }\end{array}$ & Yes & Yes & Yes \\
Promising situations & & & \\
Underlying diseases & & & \\
$\quad$ Sepsis & Yes & Yes & Yes \\
$\geq 15 \%$ BM macrophages & Yes & NE & NE \\
Recent transfusion & NE & Yes & Yes \\
Hematological malignancies and aplastic & Yes NE & NE \\
anemia & & & \\
Cause of death & Yes & Yes & NE \\
ARDS/sepsis/septic shock & & & \\
Exclusion & Yes & Yes & Yes \\
Definitive HPS/HLH &
\end{tabular}

ARDS acute respiratory distress syndrome, $B M$ bone marrow, $H L H$ hemophagocytic lymphohistiocytosis, $H P F$ high-power field, $H P S$ hemophagocytic syndrome, $N E$ not evaluated and HHH have hypercytokinemia in common, but HPS is a syndrome with defined clinical and laboratory features, one of which is the occurrence of increased histiocytes with hemophagocytosis. By contrast to HPS, the development of $\mathrm{HHH}$ is most often a multifactorial process rather than one related to a single underlying condition [2]. Therefore, $\mathrm{HHH}$ should be considered as a different disease entity from HPS. Additionally, we found a new feature of $\mathrm{HHH}$ in that the absolute number of BM histiocytes was in a similar range in the presence or absence of $\mathrm{HHH}$, indicating that the essential feature of $\mathrm{HHH}$ is the augmentation of hemophagocytic histiocytes rather than histiocytic hyperplasia. Hence, we propose a tentative definition of this disorder as "polyhemophagocytosis (PHP)" to avoid confusion with HPS (Table 7) [2, 8].

In conclusion, we found that conditions of excessive inflammation, such as hematological disorders and sepsis, appear to be the strongest initiators of HHH development. In addition, the development of $\mathrm{HHH}$ was also associated with increased IL- 6 and IL- 8 concentrations. Although further investigations of $\mathrm{HHH}$ are needed, insights into $\mathrm{HHH}$ can be gained from the analysis of data from the present study.

Acknowledgment This research was supported by Grants-in-Aid from the Ministry of Education, Culture, Sports, Science, and Technology of Japan to K. Inai, S. Noriki, and H. Naiki.

Conflicts of interest The authors declare that they have no conflict of interest.

Open Access This article is distributed under the terms of the Creative Commons Attribution License which permits any use, distribution, and reproduction in any medium, provided the original author(s) and the source are credited.

\section{References}

1. Kumar V, Abbas AK, Fausto N, Aster JC (2008) Pathologic bases of disease, 8th edn. Saunders, Philadelphia 
2. Suster S, Hilsenbeck S, Rywlin AM (1988) Reactive histiocytic hyperplasia with hemophagocytosis in hematopoietic organs: a reevaluation of the benign hemophagocytic proliferations. Hum Pathol 19:705-712

3. Rouphael NG, Talati NJ, Vaughan C, Cunningham K, Moreira R, Gould C (2007) Infections associated with haemophagocytic syndrome. Lancet Infect Dis 7:814-822

4. Janka GE (2007) Hemophagocytic syndromes. Blood Rev 21:245-253

5. Osugi Y, Hara J, Tagawa S, Takai K, Hosoi G, Matsuda Y, Ohta H, Fujisaki H, Kobayashi M, Sakata N, Kawa-Ha K, Okada S, Tawa A (1997) Cytokine production regulating Th1 and Th2 cytokines in hemophagocytic lymphohistiocytosis. Blood 89:4100-4103

6. Tang YM, Xu XJ (2011) Advances in hemophagocytic lymphohistiocytosis: pathogenesis, early diagnosis/differential diagnosis, and treatment. ScientificWorldJournal 11:697-708

7. Szyper-Kravitz M (2009) The hemophagocytic syndrome/ macrophage activation syndrome: a final common pathway of a cytokine storm. Isr Med Assoc J 11:633-634

8. Strauss R, Neureiter D, Westenburger B, Wehler M, Kirchner T, Hahn EG (2004) Multifactorial risk analysis of bone marrow histiocytic hyperplasia with hemophagocytosis in critically ill medical patients a postmortem clinicopathologic analysis. Crit Care Med 32:1316-1321

9. Hot A, Jaisson I, Girard C, French M, Durand DV, Rousset H, Ninet J (2009) Yield of bone marrow examination in diagnosing the source of fever of unknown origin. Arch Intern Med 169:2018-2023

10. Milner JD, Orekov T, Ward JM, Cheng L, Torres-Velez F, Junttila I, Sun G, Buller M, Morris SC, Finkelman FD, Paul WE (2010) Sustained IL4 exposure leads to a novel pathway for hemophagocytosis, inflammation, and tissue macrophage accumulation. Blood 116:2476-2483

11. American College of Chest Physicians/Society of Critical Care Medicine Consensus Conference: Definitions for sepsis and organ failure and guidelines for the use of innovative therapies in sepsis (1992). Crit Care Med 20:864-874.

12. Levy MM, Fink MP, Marshall JC, Abraham E, Angus D, Cook D, Cohen J, Opal SM, Vincent JL, Ramsay G, Sccm/Esicm/Accp/Ats/ Sis, (2003) $2001 \mathrm{SCCM} / \mathrm{ESICM} / \mathrm{ACCP} / \mathrm{ATS} / \mathrm{SIS}$ International Sepsis Definitions Conference. Crit Care Med 31:1250-1256

13. Kaneko T, Wada H (2011) Diagnostic criteria and laboratory tests for disseminated intravascular coagulation. J Clin Exp Hematopathol 51: $67-76$

14. MacKinnon AC, Farnworth SL, Hodkinson PS, Henderson NC, Atkinson KM, Leffler H, Nilsson UJ, Haslett C, Forbes SJ, Sethi T (2008) Regulation of alternative macrophage activation by galectin3. J Immunol 180:2650-2658

15. Inai $\mathrm{K}$, Iwasaki $\mathrm{H}$, Noriki $\mathrm{S}$, Ikegaya $\mathrm{S}$, Yamashita $\mathrm{M}$, Imamura $\mathrm{Y}$, Takimoto N, Kato H, Ueda T, Naiki H (2007) Frequent detection of multidrug-resistant pneumonia-causing bacteria in the pneumonia lung tissues of patients with hematological malignancies. Int $\mathrm{J}$ Hematol 86:225-232

16. Inai K, Noriki S, Iwasaki H (2014) Should prophylactic thrombolysis be routine in clinical practice? Evidence from an autopsy case of septicemia. BMC Clin Pathol 14:6
17. Tai K, Iwasaki H, Ikegaya S, Ueda T (2013) Minocycline modulates cytokine and chemokine production in lipopolysaccharide-stimulated THP-1 monocytic cells by inhibiting IkappaB kinase alpha/beta phosphorylation. Transl Res 161:99-109

18. Holm S (1979) A simple sequentially rejective multiple test procedure. Scan J Stat 6:65-70

19. Tang Y, Xu X, Song H, Yang S, Shi S, Wei J, Pan B, Zhao F, Liao C, Luo C (2008) Early diagnostic and prognostic significance of a specific Th1/Th2 cytokine pattern in children with haemophagocytic syndrome. Br J Haematol 143:84-91

20. Levy L, Nasereddin A, Rav-Acha M, Kedmi M, Rund D, Gatt ME (2009) Prolonged fever, hepatosplenomegaly, and pancytopenia in a 46-year-old woman. PLoS Med 6:e1000053

21. Wong KF, Chan JK (1992) Reactive hemophagocytic syndrome-a clinicopathologic study of 40 patients in an Oriental population. Am J Med 93:177-180

22. Emmenegger U, Reimers A, Frey U, Fux C, Bihl F, Semela D, Cottagnoud P, Cerny A, Spaeth PJ, Neftel KA (2002) Reactive macrophage activation syndrome: a simple screening strategy and its potential in early treatment initiation. Swiss Med Wkly 132:230-236

23. Pagano L, Caira M, Valentini CG, Posteraro B, Fianchi L (2010) Current therapeutic approaches to fungal infections in immunocompromised hematological patients. Blood Rev 24:51-61

24. Munford RS, Pugin J (2001) Normal responses to injury prevent systemic inflammation and can be immunosuppressive. Am J Respir Crit Care Med 163:316-321

25. Reichelt U, Jung R, Nierhaus A, Tsokos M (2005) Serial monitoring of interleukin-1beta, soluble interleukin-2 receptor and lipopolysaccharide binding protein levels after death: a comparative evaluation of potential postmortem markers of sepsis. Int J Legal Med 119:80-87

26. Tsokos M, Reichelt U, Jung R, Nierhaus A, Puschel K (2001) Interleukin-6 and C-reactive protein serum levels in sepsis-related fatalities during the early postmortem period. Forensic Sci Int 119: $47-56$

27. Xing L, Remick DG (2004) Neutrophils as firemen, production of anti-inflammatory mediators by neutrophils in a mixed cell environment. Cell Immunol 231:126-132

28. Bozza FA, Salluh JI, Japiassu AM, Soares M, Assis EF, Gomes RN, Bozza MT, Castro-Faria-Neto HC, Bozza PT (2007) Cytokine profiles as markers of disease severity in sepsis: a multiplex analysis. Crit Care 11:R49

29. Gomez-Jimenez J, Martin MC, Sauri R, Segura RM, Esteban F, Ruiz JC, Nuvials X, Boveda JL, Peracaula R, Salgado A (1995) Interleukin-10 and the monocyte/macrophage-induced inflammatory response in septic shock. J Infect Dis 171:472-475

30. Waugh DJ, Wilson C (2008) The interleukin-8 pathway in cancer. Clin Cancer Res 14:6735-6741

31. Schwabe M, Hartert AM, Bertz H, Finke J (2004) Interleukin-8, neutropenia, and graft failure in human stem cell transplantation. Transplantation 78:1086-1088 